\title{
In Vivo Flow Dynamics of the Total Cavopulmonary Connection from Three-dimensional Multislice Magnetic Resonance Imaging
}

\author{
Shiva Sharma \\ Children's Heart Center
}

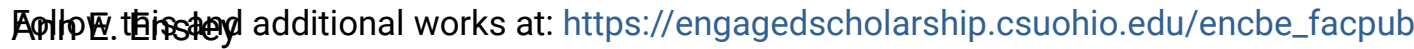

¿ropia Institute of Technology

Katwertweskopkisto this work benefit you? Let us know!

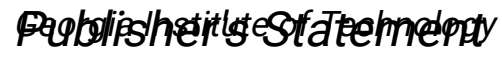

ElTJCE. this is the author's version of a work that was accepted for publication in Annals of

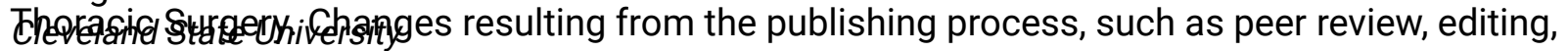
corrections, structural formatting, and other quality control mechanisms may not be reflected in Timothy M. Healy Georgia Institute of Techno may have been made to this work since it was submitted for publication. A definitive version was subsequently published in Annals of Thoracic Surgery, 71,

3, (March 2001) DOI 10.1016/S0003-4975(00)02517-0

See next page for additional authors

\section{Original Citation}

Sharma S, Ensley AE, Hopkins K, Chatzimavroudis GP, Healy TM, Tam VKH, Kanter KR, Yoganathan AP. In vivo flow dynamics of the total cavopulmonary connection from three-dimensional multislice magnetic resonance imaging. Ann Thorac Surg. 2001;71:889-898.

\section{Repository Citation}

Sharma, Shiva; Ensley, Ann E.; Hopkins, Katherine; Chatzimavroudis, George P.; Healy, Timothy M.; Tam, Vincent K.H.; Kanter, Kirk R.; and Yoganathan, Ajit P., "In Vivo Flow Dynamics of the Total Cavopulmonary Connection from Three-dimensional Multislice Magnetic Resonance Imaging" (2001). Chemical \& Biomedical Engineering Faculty Publications. 105.

https://engagedscholarship.csuohio.edu/encbe_facpub/105

This Article is brought to you for free and open access by the Chemical \& Biomedical Engineering Department at EngagedScholarship@CSU. It has been accepted for inclusion in Chemical \& Biomedical Engineering Faculty Publications by an authorized administrator of EngagedScholarship@CSU. For more information, please contact library.es@csuohio.edu. 


\section{Authors}

Shiva Sharma, Ann E. Ensley, Katherine Hopkins, George P. Chatzimavroudis, Timothy M. Healy, Vincent K.H. Tam, Kirk R. Kanter, and Ajit P. Yoganathan 


\title{
In Vivo Flow Dynamics of the Total Cavopulmonary Connection From Three- Dimensional Multislice Magnetic Resonance Imaging
}

\author{
Shiva Sharma, MD, Ann E. Ensley, BS, Katharine Hopkins, MD, \\ George P. Chatzimavroudis, PhD, Timothy M. Healy, BS, Vincent K. H. Tam, MD, \\ Kirk R. Kanter, MD, and Ajit P. Yoganathan, PhD
}

The Children's Heart Center, Atlanta, Georgia, Department of Cardiothoracic Surgery at Emory University School of Medicine, Atlanta, Georgia, School of Biomedical Engineering, Georgia Institute of Technology, Atlanta, Georgia, and Department of Chemical Engineering, Cleveland State University, Cleveland, Ohio

Background. The total cavopulmonary connection (TCPC) design continues to be refined on the basis of flow analysis at the connection site. These refinements are of importance for myocardial energy conservation in the univentricular supported circulation. In vivo magnetic resonance phase contrast imaging provides semiquantitative flow visualization information. The purpose of this study was to understand the in vivo TCPC flow characteristics obtained by magnetic resonance phase contrast imaging and compare the results with our previous in vitro TCPC flow experiments in an effort to further refine TCPC surgical design.

Methods. Twelve patients with TCPC underwent sedated three-dimensional, multislice magnetic resonance phase contrast imaging. Seven patients had intraatrial lateral tunnel TCPC and 5 had extracardiac TCPC.

Results. In all patients in both groups a disordered flow pattern was observed in the inferior caval portion of the TCPC. Flow at the TCPC site appeared to be determined by connection geometry, being streamlined at the superior

$\mathrm{C}$ hildren with an increasingly wide variety of congenital heart defects are undergoing the univentricular supported total cavopulmonary connection (TCPC) operation as their definitive repair. Many variables determine the clinical outcome of these patients $[1,2]$. One of the variables that allows some degree of control is the surgically created geometry of the TCPC. Currently, several geometric designs of TCPC are being practiced in different institutions [3-5]. A major consideration in designing the TCPC is to optimize flow energetics in the univentricular-supported circulation as that would lower systemic venous pressures and also decrease the work load of the ventricle. Several in vitro and in vivo studies have compared the atriopulmonary connection with the TCPC and have demonstrated vena cava-pulmonary junction when the superior vena cava was offset and flared toward the left pulmonary artery. Without caval offset, intense swirling and dominance of superior vena caval flow was observed. In TCPC with bilateral superior vena cavae, the flow patterns observed included secondary vortices, a central stagnation point, and influx of the superior vena cava flow into the inferior caval conduit. A comparative analysis of in vivo flow and our previous in vitro flow data from glass model prototypes of TCPC demonstrated significant similarities in flow disturbances. Three-dimensional magnetic resonance phase contrast imaging in multiple coronal planes enabled a comprehensive semiquantitative flow analysis. The data are presented in traditional instantaneous images and in animated format for interactive display of the flow dynamics.

Conclusions. Flow in the inferior caval portion of the TCPC is disordered, and the TCPC geometry determines flow characteristics.

improvement in flow energetics in the latter design [6-9]. However, the flow dynamics of the different types of TCPC designs have not been described in any in vivo study.

In our in vitro experiments we studied three different designs of the TCPC with quantitative assessment of energy losses and correlated the losses with flow visualization $[7,10,11]$. This gave information regarding connections that would conserve energy and those that were particularly detrimental in this regard. On the basis of this information, we modified our TCPC design. Cur- 


\begin{tabular}{|c|c|c|c|c|c|}
\hline Patient No. & General Diagnosis & $\begin{array}{c}\text { TCPC } \\
\text { Connection }\end{array}$ & Age at Scan & $\begin{array}{l}\text { Time } \\
\text { Elapsed }\end{array}$ & $\begin{array}{c}\text { Fenestration } \\
\text { Status }\end{array}$ \\
\hline 1 & TA, D-TGA & IA & $76 \mathrm{mo}$ & $48 \mathrm{mo}$ & Closed \\
\hline 2 & DORV, Sub PS, Bilateral SVC & IA & 99 mo & $70 \mathrm{mo}$ & Closed \\
\hline 3 & HLHS & EC & $23 \mathrm{mo}$ & $2 \mathrm{mo}$ & Open \\
\hline 4 & TA & IA & $103 \mathrm{mo}$ & $60 \mathrm{mo}$ & Closed \\
\hline 5 & Unbalanced AVSD & EC & $36 \mathrm{mo}$ & $8 \mathrm{mo}$ & Open \\
\hline 6 & DILV w/Vent Inversion & IA & 224 mo & $90 \mathrm{mo}$ & Closed \\
\hline 7 & DILV, D-TGA, Bilateral SVC & IA & $51 \mathrm{mo}$ & $24 \mathrm{mo}$ & Open \\
\hline 8 & TA & IA & $104 \mathrm{mo}$ & $66 \mathrm{mo}$ & Open \\
\hline 9 & $\begin{array}{l}\text { Ventricular Inversion } \\
\text { Pulmonary Atresia }\end{array}$ & IA & $111 \mathrm{mo}$ & $72 \mathrm{mo}$ & Closed \\
\hline 10 & $\begin{array}{l}\text { Interrupted Ao Arch } \\
\text { Sub AS }\end{array}$ & EC & $36 \mathrm{mo}$ & $11 \mathrm{mo}$ & Open \\
\hline 11 & HLHS & $\mathrm{EC}$ & $21 \mathrm{mo}$ & $3 \mathrm{mo}$ & Open \\
\hline 12 & HLHS & EC & $24 \mathrm{mo}$ & $3 \mathrm{mo}$ & Open \\
\hline
\end{tabular}

AS = aortic stenosis; $\quad$ AVSD = atrioventricular septal defect; $\quad \mathrm{EC}=$ extracardiac connection; $\quad \mathrm{IA}=$ intraatrial tunnel connection; $\quad \mathrm{D}-\mathrm{TGA}=$ D-transposition of great arteries; $\quad$ HLHS = hypoplastic left heart syndrome; $\quad$ TA = tricuspid atresia; $\quad$ DILV = double inlet left ventricle; DORV = double outlet right ventricle; $\quad$ SVC = superior vena cava; $\quad$ PS = pulmonic stenosis.

rently, at our institution, two types of TCPC are performed by us: A lateral intraatrial tunnel and an extracardiac conduit.

The flow characteristics within the TCPC are time varying and likely to be spatially complex. Magnetic resonance phase contrast imaging has become established as the only method available for providing timedependent velocity mapping of flow, which gives both direction and magnitude of flow. It is unique in its ability to acquire three-dimensional velocity encoding. When such acquisition is done in multiple slices in the coronal planes through the TCPC, a reasonably complete representation of flow characteristics is obtained.

The purpose of this study was to define the in vivo flow characteristics of the above mentioned two types of TCPC by magnetic resonance phase contrast imaging and compare the results with our previous in vitro flow analysis for possible further energy conserving refinements of TCPC design.

\section{Material and Methods}

\section{Patients}

From 20 patients who underwent magnetic resonance scans, 12 patients had complete studies and form the study group. In 8 of 20 patients, studies were excluded for movement artifact due to patient awakening [3], artifact from coils in systemic pulmonary collaterals [1], insufficient scan time [3], and a technical masking error [1]. The study protocol was approved by the Institutional Review Board of Emory University School of Medicine and informed consent was obtained from parents. The studies were fully funded by American Heart Association (Georgia Affiliate) Grant for the study period from July 1996 to July 1999.

The patients were operated on by surgeons KRK and VKHT and all patients had undergone a previous bidi- rectional Glenn anastomosis. Table 1 describes the basic diagnosis of patients studied, the age at time of scan, elapsed time between operation and scan, the type of conduit used, and the status of the fenestration. Two patients with bilateral superior vena cavae (SVC) underwent bilateral Glenn anastomoses. All patients operated on by KRK underwent Intraatrial lateral TCPC and the patients operated on by VKHT underwent an extracardiac TCPC and these conduits will be referred to as inferior caval conduits (ICC). The median time elapsed between date of operation and date of scan was 13 months (Table 1). All patients in the study group had undergone echocardiography within 6 months of the scan and the status of the fenestrations in the inferior caval conduit was determined by the echocardiogram.

\section{Magnetic Resonance Imaging Protocol}

The magnetic resonance images used in this study were acquired using a 1.5-T whole-body clinical scanner (GE Medical Systems, Milwaukee, WI). Coronal and axial scout images were used to localize the TCPC site in addition to the use of a standard clinical T1 spin-echo acquisition. These images served as a reference from which coronal slices encompassing the connection could be located for flow quantification.

Once slice locations were determined, velocity data were acquired using a cine PC gradient-echo pulse sequence. Three to five contiguous coronal slices with a thickness of $5 \mathrm{~mm}$ were acquired during this phase of the examination. Velocity was encoded in each slice along three principal directions: superior-inferior, right-left, and anterior-posterior. Respiratory and retrospective electrocardiographic gating were used to acquire 16 phases in each axis during the cardiac cycle. This was done with a temporal resolution of 31 milliseconds, Respiratory gating was such that acquisitions were done at the nadir of respiratory excursion. Imaging data were 
Table 2. Imaging Parameters for the Cine PC Gradient-Echo

\begin{tabular}{lc} 
Velocity Encoding Acquisitions & \\
\hline Pulse Sequence & Cine PC Gradient-Echo \\
\hline Number of slices & $1-5$ \\
Slice thickness $(\mathrm{mm})$ & 5 \\
Field of view $(\mathrm{mm} \times \mathrm{mm})$ & $200 \times 200$ \\
Flip angle $\left({ }^{\circ}\right)$ & 35 \\
Repetition time $(\mathrm{ms})$ & 23 \\
Echo time $(\mathrm{ms})$ & 6.2 \\
Number of signals averaged & 2 \\
Matrix size & $256 \times 256$ \\
Velocity encoding value $(\mathrm{cm} / \mathrm{s})$ & 70
\end{tabular}

held fixed for each acquisition; these values are summarized in Table 2. The total examination time was approximately 75 minutes. To minimize imaging artifacts associated with movement of patients during the acquisition, the patients less than 10 years of age were sedated with intravenous nembutal (4 to $6 \mathrm{mg} / \mathrm{kg}$ ). The patients were continuously monitored by pulse oximetry and electrocardiogram. None of the patients demonstrated any significant arterial oxygen desaturation or any bradycardia.

After the examination, the images were downloaded from the scanner to a Sun SPARC station (Sun Microsystems, Mountain View, CA). Image masking, data translation, and visualization were then performed on a Windows-based PC (Toshiba American Information Systems, Inc, Irvine, CA). Transform (Fortner Research, LLC, Sterling, VA) software was used to select the region of interest, creating a mask of that region, and filtering the data using this region of interest mask. The region of interest of the TCPC, including SVC, ICC, and pulmonary arteries (PA) was outlined manually using the magnitude images that clearly showed the varying contrast of the TCPC connection. A series of macros, written in the Georgia Tech Cardiovascular Fluid Mechanics Laboratory were then used to automate the system by creating a mask based on the outlined region of interest and filtering the remaining data to keep only the velocity information inside the TCPC. The resulting files from Transform were in format and using the method described by Healy and colleagues [12] were loaded into Tecplot (Amtec Engineering, Bellevue, WA) for visualization. In 4 patients the coronal plane dataset was cut in three sagittal planes along the lines shown in the inset of Figure 1. Figure 1 itself represents the sagittal cuts that are derived from patient 1 and represent the orthogonal views to Figure 2.

\section{Results}

\section{Anatomy of Connection Types}

Five patients had an extracardiac TCPC. All of these patients had undergone a previous bidirectional Glenn anastomosis in which the SVC was preferentially flared

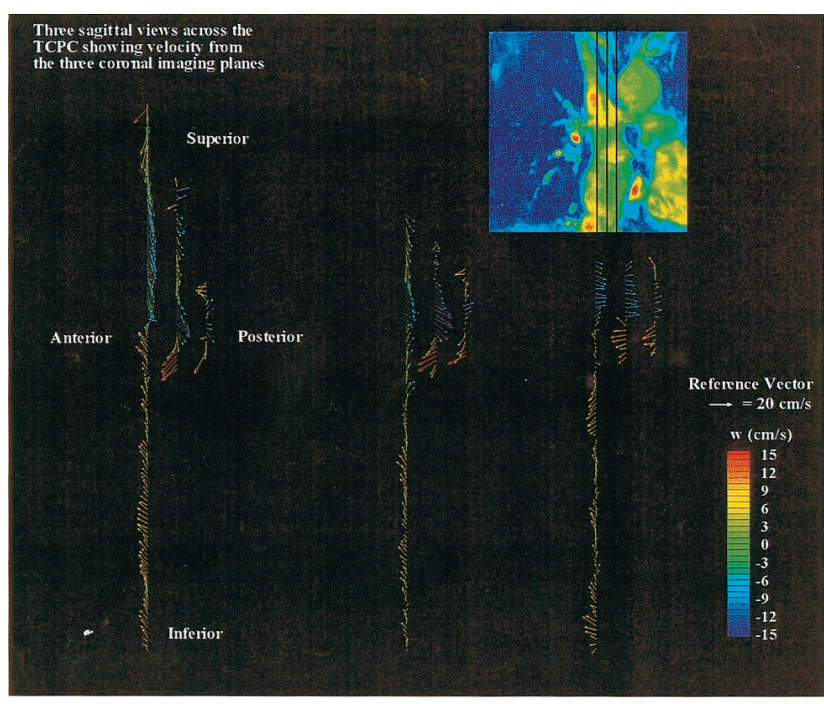

Fig 1. Vector maps from sagittal views cut along the lines shown in the inset of the coronal plane of patient 1 . The maps display the helical flow set up at the connection site with the anterior-posterior vectors profiled clearly.

open toward the left PA. The extracardiac conduit consisted of aortic homograft material and was curved on the right and anterior portion of the right atrium and con-

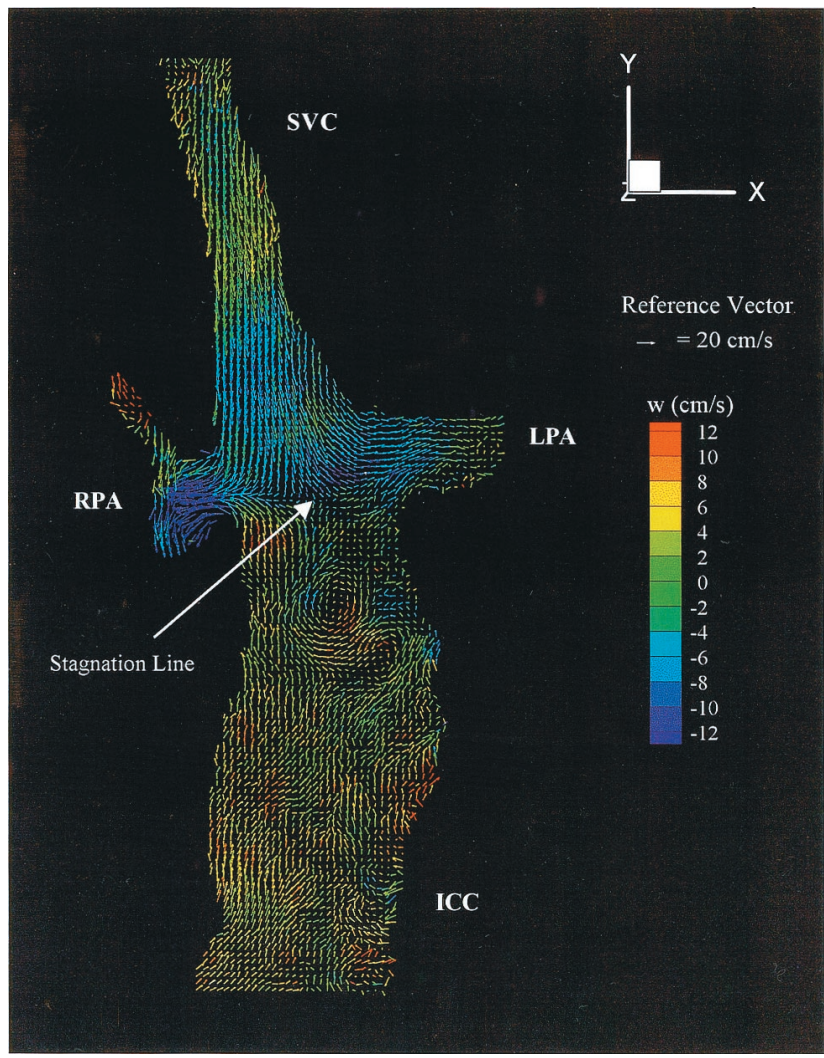

Fig 2. A vector map in a coronal slice from the same patient as in Figure 6 (Patient 1). Note the disordered flow in the inferior caval conduit. The increased superior vena caval velocity and its dominance is demonstrated by the inferior displacement of the stagnation line. (ICC = inferior caval conduits; LPA = left pulmonary artery; $\mathrm{RPA}=$ right pulmonary artery; $\mathrm{SVC}=$ superior vena cava.) 


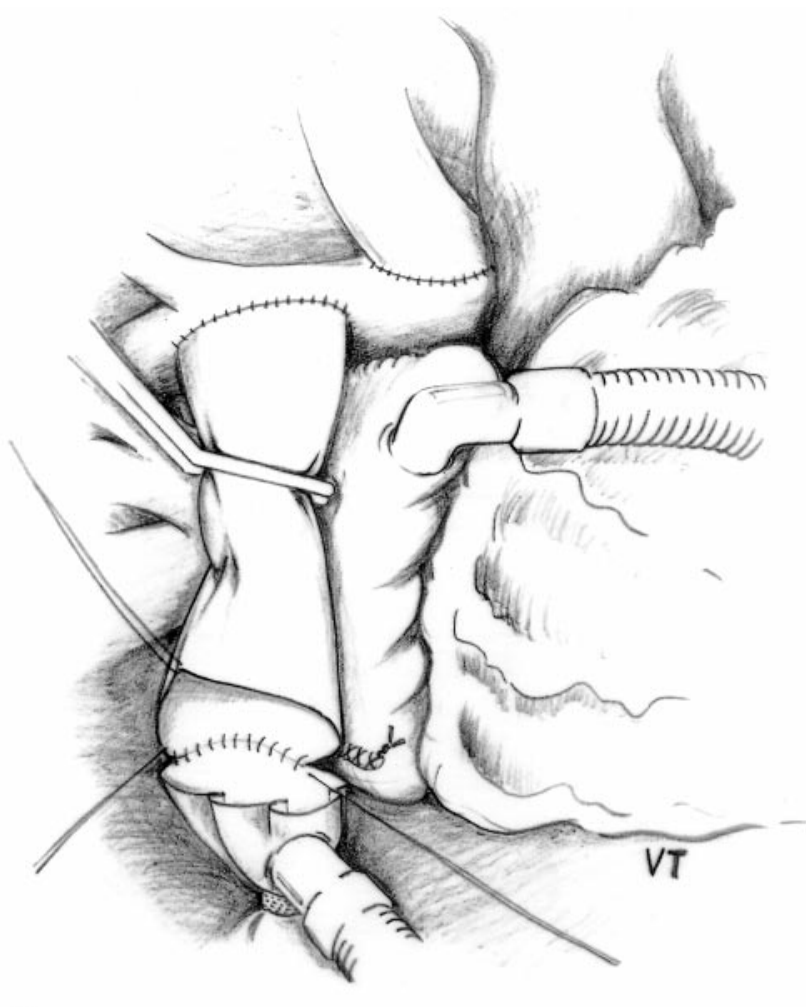

A

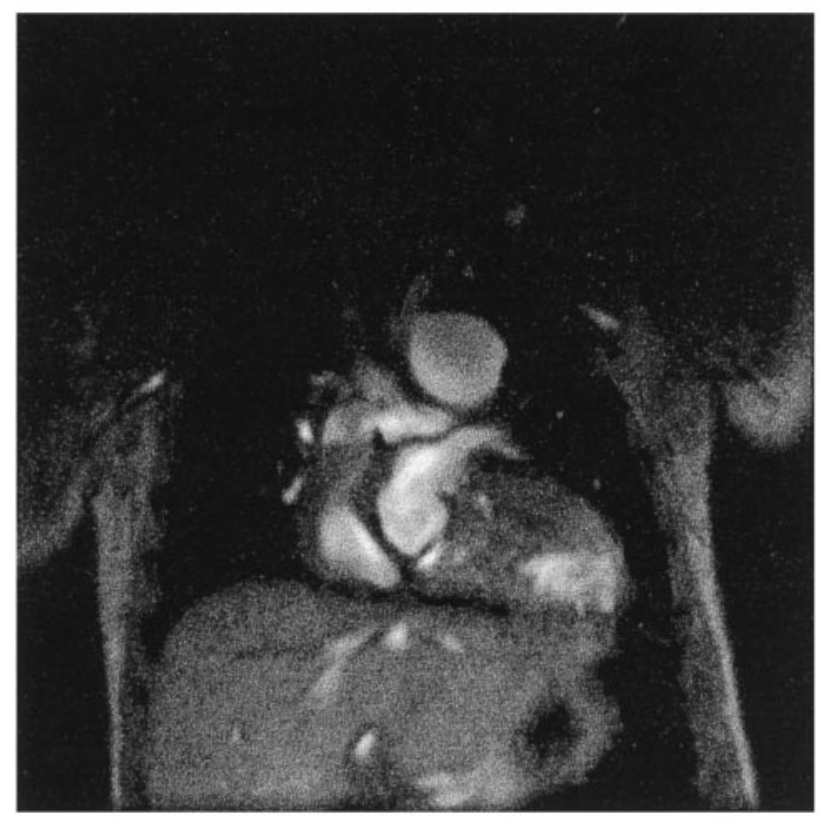

B

Fig 3. (A) Schematic of extracardiac total cavopulmonary connection with the superior vena caval offset and flared toward the left pulmonary artery. (B) Magnetic resonance magnitude image of a coronal slice from an extracardiac type of total cavopulmonary connection. Note the waist at the midinferior caval conduit level. This figure corresponds with Figure 5 and Patient 11.

nected to the right PA in a bilaterally flared open design (Fig 3).

Seven patients had an intraatrial lateral tunnel TCPC. In

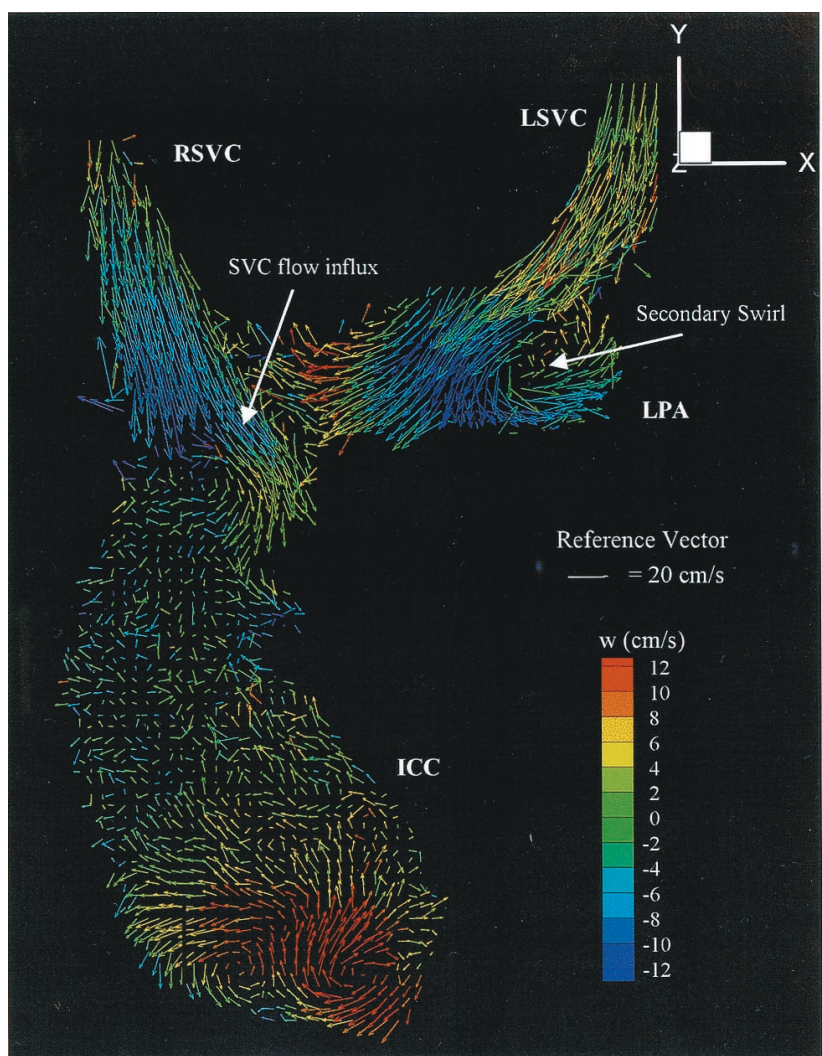

Fig 4. A vector map in a coronal slice from a patient with bilateral superior vena cava (Patient 7). The picture demonstrated a complex anteriorly directed swirl in the lower inferior caval conduit from which an area of acceleration emerges. Note superior vena caval flow influx into inferior caval conduit and secondary swirl in the left pulmonary artery. (ICC $=$ inferior caval conduits; LPA $=$ left pulmonary artery; $\mathrm{LSVC}=$ left superior vena cava; $\mathrm{RSVC}=$ right superior vena cava; $\mathrm{SVC}=$ superior vena cava.)

these patients the SVC connection with the right PA was flared but not preferentially toward either side. The ICC was brought intraatrially and connected to the left of the SVC-right PA anastomosis and flared open in all directions. Two patients had bilateral SVCs. The material used for the lower part of the ICC was a hemicylinderical Gore-Tex graft (W. L. Gore \& Associates, Flagstaff, AZ) and at the pulmonary artery end the patient's right atrial appendage was used to complete the connection.

The 2 patients with bilateral SVCs formed a subtype of TCPC connection. These patients did not have any additional systemic or pulmonary venous anomalies to suggest heterotaxy syndrome. Both patients had an intraatrial ICC that connected between the two superior caval connections and little closer to the right SVC (Fig 4).

The results are divided into the flow observations made at three arbitrary components of the TCPC, that is, the superior cavae and inferior caval conduit, the cavopulmonary connection site, and the branch pulmonary arteries. These components were difficult to visualize in any single coronal slice and in each patient multiple slices were viewed to completely analyze the TCPC flow dynamics. 


\section{Inferior Caval Conduit and SVC Flow}

Figures 2, 4, and 5 are vector maps of coronal slices that demonstrate SVC and ICC flow. Figures 2 and 4 (patients 1 and 7, respectively) represent examples of the intraatrial type of TCPC and Figure 5 is an extracardiac type of TCPC (patient 11). The flow depicted is representative of flows seen in the ICC and SVC of the total patient group. Each figure has a vector scale in centimeters per second with assignment of red hues to the component of velocity directed anteriorly and blue hues to that directed posteriorly. Figure 1 shows the three sagittal views of the TCPC as obtained by slicing the coronal planes along the lines shown in the inset. We observed that the ICC flow had overall lower velocities (shorter arrow lengths) than the SVC flow. The SVC flow was observed to be smooth and streamlined in all patients. It appeared to be posteriorly directed (Fig 1). The ICC flow, as observed in Figures 1, 2, 4, and 5, was disordered in all patients. Circular swirling flow was noted in several areas. Multiple stagnation points with very small vectors were observed in random places along the pathway (arrows). Figure 5 demonstrates an area of increased velocity flow directed anteriorly in the lower portion of the ICC. It appears to originate from the region of hepatic veins.

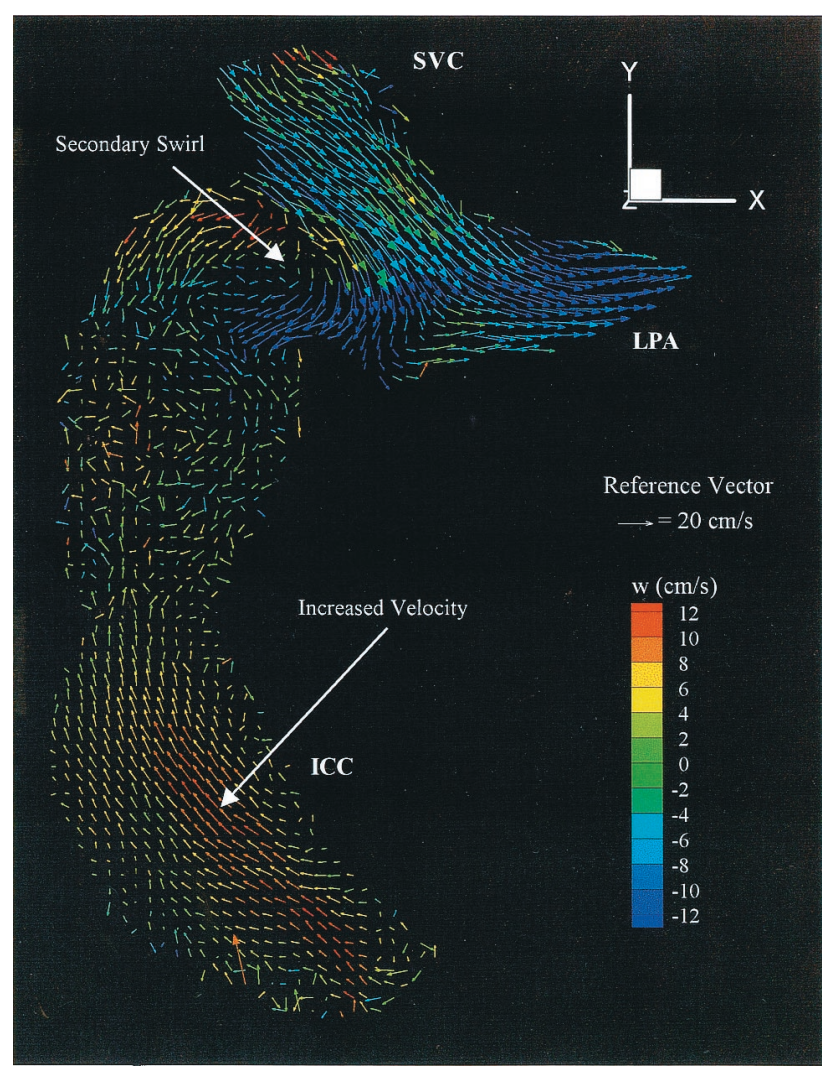

Fig 5. A vector map in a coronal slice from the patient in Figure $3 B$ (Patient 11). Note the increased velocity flow in the inferior caval conduit. Streamlined superior vena cava and left pulmonary artery flow can be seen as well as a secondary swirl. (ICC = inferior caval conduits; $\mathrm{LPA}=$ left pulmonary artery; $\mathrm{SVC}=$ superior vena cava.)

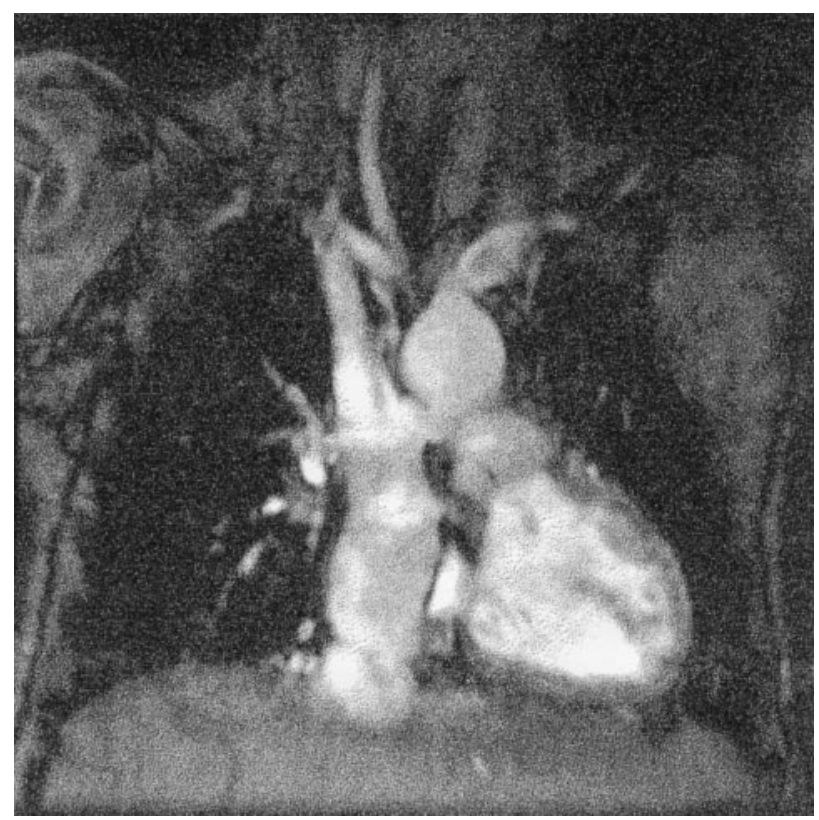

Fig 6. Magnetic resonance magnitude image taken from coronal slice of a patient with an intraatrial type total cavopulmonary connection with no offset. This figure corresponds with

Figure 2 and Figure 1 inset.

This type of flow was observed in 7 of 12 patients. In 1 patient a reversal of flow into the hepatic vein was observed in diastole for the duration of two phases.

The animation displays all 16 phases that were acquired. The high velocity flow in the lower ICC appeared to originate from the region of the hepatic veins and was observed to be phasic, present in the mid-phases corresponding with late systole and early diastole. This is best seen in the animation of Figure 5. The animation of Figure 4 also shows a similar area of increased velocity directed anteriorly. However, it appears more complex as it appears to originate from a circular swirl. The stagnation points in the patients did not appear to have any fixed spatial or temporal relationship. Details of circular swirling can be observed. We were unable to localize the fenestration in patients in whom we believed it to be open by echocardiography. In all patients the disordered flow appeared to be present throughout the conduit and was not more pronounced near the walls. In Figure 5 a waist is noted at the midconduit level corresponding to the waist noted from the magnitude image (Fig 3B). Flow upstream from this region appears more uniform, albeit in later phases; it is also disordered. However, flow downstream from this junction appears more disordered.

\section{Flow at Connection Site}

NO OFFSET. In 1 patient, with no offset (Fig 2 and Fig 6), there was a direct collision of caval flow with intense interaction. At the site of collision, however, a linear region of low velocity flow was noted. This is referred to as the line of stagnation and is better depicted in the Figure 2 animation. The dominance of SVC velocity was evident by a stagnation line that was closer to the ICC 


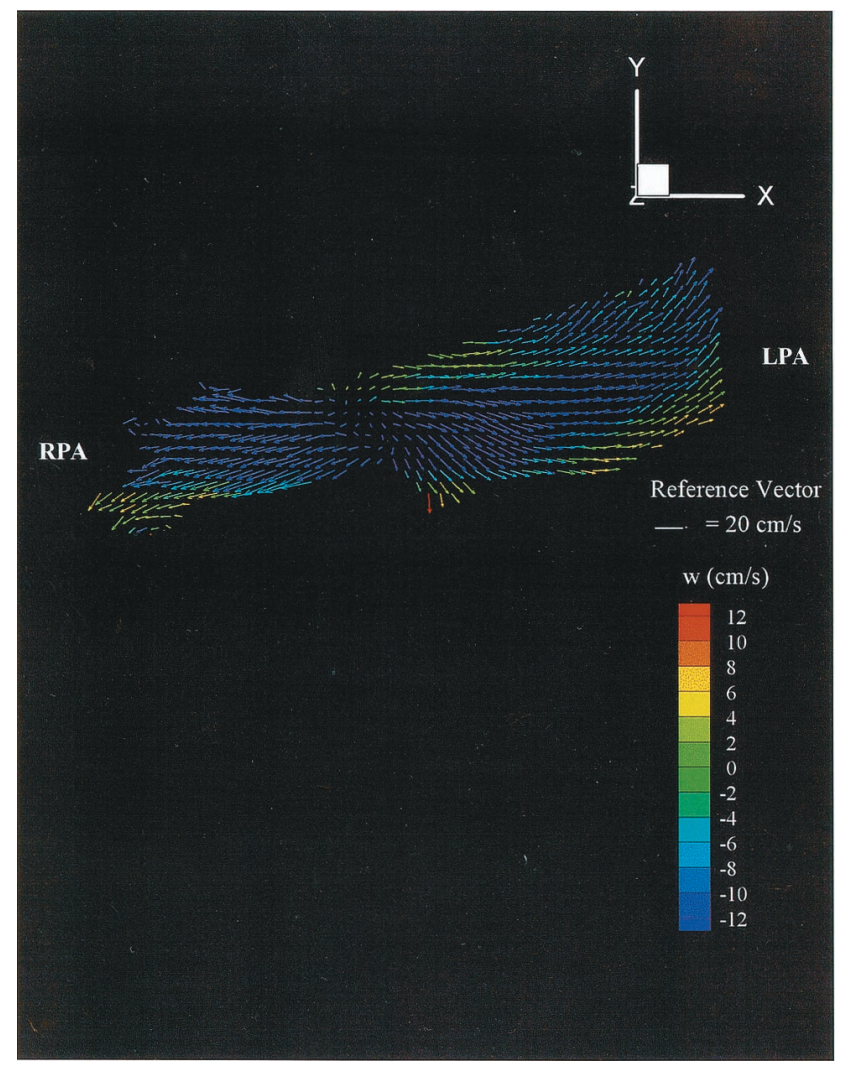

Fig 7. A vector map in the coronal plane of the branch pulmonary arteries demonstrating posteriorly directed flow. (LPA = left pulmonary artery; RPA = right pulmonary artery.)

end (arrow). Adjacent to the collision site an anteriorposterior/inferior-superior swirl was observed and was best seen in the sagittal view (Fig 1). At this site, the flow from the SVC was first directed posteriorly and then it curved around to turn inferior and then anteriorly. This pattern was noted throughout the length of the connection site and gave rise to a helical flow pattern.

In the animation (Fig 2 animation) the dominance of SVC flow is fully appreciated with the line of stagnation displaced more inferiorly. The nature of the intense caval interaction was better defined. The circular flow pattern can now be fully observed with a rotational axis that lies predominantly in the anterior-posterior and superiorinferior planes. This feature is easier to see in the orthogonal plane to Figure 2, which is represented in Figure 1 animation.

WITH OFFSET. Figure 5 is a representative case of caval offset at the connection and shows the SVC offset and flared preferentially toward the left PA and the extracardiac ICC brought to the right of this junction. Streamlined flow with unidirectionality of arrows was noted toward the left PA from the SVC in all 5 patients. In 3 patients, flow from the right side of the SVC turned toward the right PA to set up a swirling secondary flow in the region of offset between the two caval flows. From this swirling, mixing of caval flows was evident. The patients with intraatrial type of TCPC (patients $4,6,8,9$ ) who had their intraatrial ICC brought to the left of the SVC connection site and flared open, demonstrated relatively smooth flow at connection site, albeit with secondary circular swirls.

Figure 5 animation demonstrates that the swirling vortex at the offset junction appears to have a predominant right-left and superior-inferior rotational axis. In this animation a smaller swirl is noted in the inferior portion of the PA opposite the SVC connection. This swirl possibly occupies the site of the main PA stump and appears to cushion the SVC flow that splits toward the right $\mathrm{PA}$.

BILATERAL SVC. Figure 4 is a vector map that demonstrates the TCPC with bilateral SVC. In this instantaneous phase, the dominant SVC flow is noted to influx into the ICC. An area of circular swirling is observed at the proximal left PA just lateral to the left SVC. The ICC appears dilated and disordered flow is observed in it.

In the patients with bilateral SVC, the animation alone gave a full appreciation of the rhythmic alternation of flows to the PAs from the two SVCs and the ICC, each of which lasts about three phases (Fig 4 animation). The central stagnation is observed only in early phases. Secondary circular swirling lateral to the left PA is observed to be phasic. There is clear demonstration of ICC flow split to both PAs with the appearance of a more dominant flow toward the left PA as compared to the split toward the right PA.

BRANCH PA FLOW. Figure 7 shows the proximal branch PAs, which were located in the posterior slice of our patients. Left PA flow appeared to be streamlined in all 5 patients in whom the SVC was flared toward the left PA (Fig 5). Localized swirling flows were noted in both branch PAs in patients with bilateral SVCs (Fig 4). The biphasic nature of flow in the PA branches was readily seen in the animation where flow was observed to slow considerably in the midportion of the phases. During the phase of slowing a helical pattern of swirling was observed.

\section{Comment}

Many institutions have achieved high early survival results after the TCPC $[1,3]$. Attention has now been directed at improving the functional outcome of children after the TCPC chiefly by optimizing the surgical design of the TCPC to conserve energy. It was de Leval's landmark in vitro experimental work that first demonstrated energy conservation in the TCPC as opposed to the atriopulmonary connection [6]. Subsequently, an in vivo magnetic resonance imaging study has also demonstrated improved flow dynamics in the TCPC over the atriopulmonary connection [9]. In a recent study, computational modeling of different types of TCPC demonstrated that offsetting of the caval connection was efficient [13]. Paralleling the in vitro studies and learning from the insights therein, many institutions have continued to modify the TCPC design. In our institution, two predominant types of TCPC are performed. In both types, offset- 
ting and flaring is done at the connection site and these modifications were significantly influenced by the findings of our in vitro experiments.

To date, a comprehensive analysis of the flow characteristics in different designs of TCPC has not been made. Magnetic resonance phase contrast imaging-derived flow visualization offers interactive and intuitive appreciation of flow characteristics and lends itself to comparison with in vitro flow visualization. We observed flow characteristics that have not been described thus far.

\section{Inferior Caval Conduit Flow}

Surprisingly, we found a significantly disordered flow pattern in the ICC of all our patients. This flow pattern appeared to be independent of type of connection, presence or absence of fenestration, or type of graft material used. Our findings differ from Fogel and colleagues [14] who, using bolus tagging magnetic resonance imaging methods, found no evidence of disordered flow anywhere in the systemic venous pathway. However, they did note the highest velocity as well as the maximum variation in velocity at the midbaffle level of the inferior caval pathway and speculated several causes for this variation in flow velocity, including the effects of motion of nearby cardiac structures, the effects of respiration, and the effects of synthetic material. Although we agree with these speculations, other possibilities exist. Larado and associates [15] have studied the effect of inferior vena cava to extracardiac conduit diameter ratio in their in vitro TCPC animal model. They measured 13\% higher energy dissipation in the models with conduit diameter 1.5 inferior vena cava than compared to 1.0 IVC and attributed this to flow expansion effects that cause flow separation and loss of streamlined flow. Our observations of disordered ICC flow could be partially explained by the same phenomenon as some of our patients demonstrated that the ICC was larger than the IVC. However, this was not systematically studied by us as it was not anticipated at the time of scan acquisition. Nevertheless, our study does support the following additional speculations of disordered ICC flow.

1. The ICC did not appear to be a uniform structure as is evident from Figures 2, 4, and 5. In Figure 5, the disordered flow appeared to be related to the waist at the midbaffle level.

2. A region of increased flow velocity was observed in the upstream portion of ICC in several patients and appeared to originate from the hepatic veins. A recent study concluded that hepatic venous flow in TCPC patients is immensely augmented in inspiration and it is possible that the disordered ICC flow observed in our study is also due to this phenomenon [16]. At the superior end of the connection, SVC influx into the conduit was noted in 3 patients despite offsetting of the SVC. In 2 of these patients there were bilateral SVCs. The observation of SVC flow influx into the ICC, despite offsetting, underscores the need to understand offsetting and flaring more fully and will be discussed later. It appears

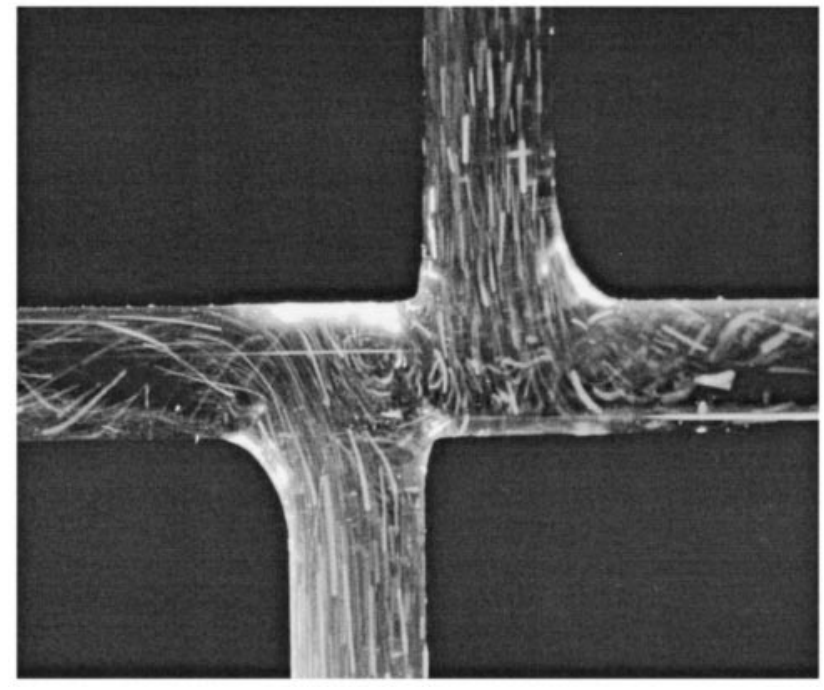

Fig 8. Particle flow visualization in the flared and 14-mm offset in vitro total cavopulmonary connection glass model. The superior vena cava-to-inferior caval conduit flow ratio was $40: 60$ with $70 \%$ of total flow (4 L/min) directed to the right pulmonary artery. A swirl is noted in the pulmonary artery between the two caval offsets.

that the influxes at both ends of ICC contributed to the overall disordered appearance of flow in some patients.

3. There appeared to be no correlation between the presence or absence of fenestration and ICC flow disturbances. In fact, the fenestration could not be localized in any of our patients by magnetic resonance phase contrast imaging and was documented by echocardiography alone. However, flow disturbances are likely to be set up by narrowed exits like fenestrations and may have played a role in forming the disordered flow of some patients.

Irrespective of the cause, we believe the disordered flow in ICC is likely to cause significant energy loss and needs further elucidation, including a detailed flow analysis of the hepatic vein-IVC conduit junction.

\section{Relationship of Connection Geometry and Flow}

A number of different caval connections were studied and a significant relationship emerged between the geometry of connection and flow. When the cavae were not offset from each other, intense caval flow interaction was observed. In this interaction the dominant flow was from the SVC. This is a consequence of the smaller size of the SVC compared to the ICC. Although, the ICC contributes about $60 \%$ of the flow to the connection [3], its larger size reduces the velocity required to deliver this flow and hence there is a lower kinetic energy per unit mass of blood in it than is in the SVC. When the SVC was flared preferentially toward the left PA and ICC toward the right PA the interaction was less, although secondary swirls were noted. When the opposite type of flaring and offset was done as in the intraatrial type of TCPC, again the flow interaction was less. When the SVC was flared in 


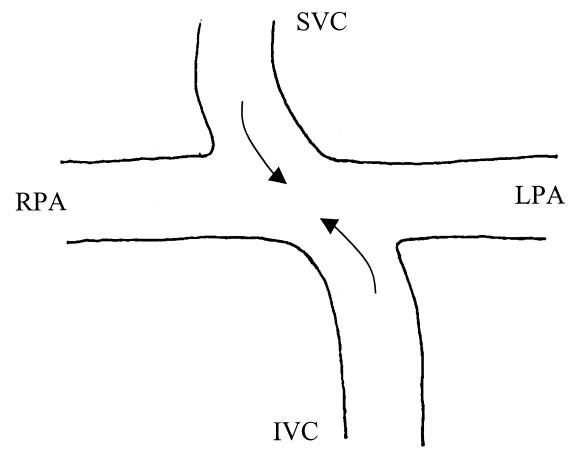

A

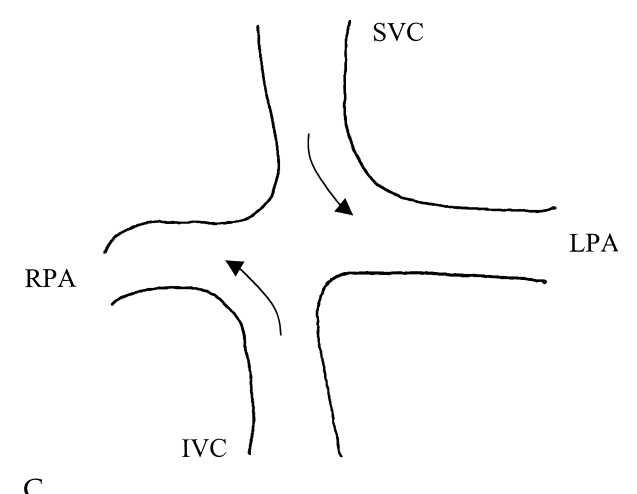

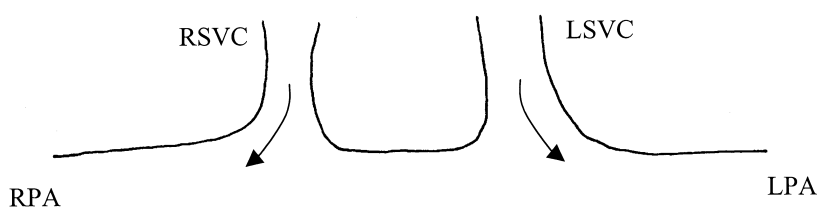

Fig 9. Schematics of total cavopulmonary connections that are desirable and undesirable. (IVC = inferior vena cava; $\mathrm{LPA}=$ left pulmonary artery; LSVC = left superior vena cava; RPA = right pulmonary artery; $\mathrm{RSVC}=$ right superior vena cava; $\mathrm{SVC}=$ superior vena cava.) a manner that directed flow toward the ICC, influx of SVC flow into the ICC resulted in disordered flow. These observations underscore the importance of connection geometry in conserving energy as is borne out by our in vitro studies of similar connection geometry [11].

\section{Branch Pulmonary Artery Flow}

The branch PAs by virtue of lying in different planes were difficult to image completely. However, the proximal branches were visible and demonstrated secondary swirls that were most likely generated from the connection site. Helical flow patterns noted in our in vitro study could not be observed in vivo in the branch PAs. However, at the connection site of the zero offset patient such helical patterns were observed and we believe that these would be present at least to some extent in the branches, as the genesis of helical flow patterns is the caval interaction of flow. Morgan and colleagues [17] have described circular swirling patterns in the branch PAs of their TCPC patients. Their analysis was performed in the cross-sectional plane of the branch PAs and perhaps it is a more sensitive method for analyzing branch PA flow. Branch PA flow observed in our study was biphasic and agrees with other studies in this regard [18, 19].

\section{Comparative Analysis of In Vivo and In Vitro Flow Patterns}

The range of in vitro glass models studied by us included designs that resembled the geometry of TCPC that are done at our institution and thus the in vivo and in vitro flow dynamics could be compared. This was also enabled by our ability to visualize flow by both methods (Fig 8).
The flow patterns observed by MRCPI appeared similar in many regards to those observed in our glass model experiments. When the SVC was flared toward the left PA, streamlined flow in the SVC remained streamlined in the left PA. This was observed by both methods (Figs 5 and 8). Flow appeared to split off the SVC toward the right PA in both flow visualizations. A circular swirl in the region of the offset was clearly observed by both methods.

When the cavae were not offset but flared, there was intense caval flow interaction producing a circular anterior-posterior swirl that extended toward the PAs. This was more noticeable in the sagittal views (Fig 1). Similar circular swirls were noted in the geometry matched in vitro model. Morgan and colleagues [17], who described similar circular swirls in cross-sectional views of the PA, calculated high circumferential shear stresses due to this swirling motion and speculated the effects of this on the cell lining. Although our study did not measure shear stresses, our in vitro data quantified high-energy losses in the "zero" offset TCPC design. From hemodynamic principles, we know that large shearing stresses lead to energy losses and thus from our data it can be inferred that energy losses are likely to be significant in the TCPC without offset.

\section{Animation}

The in vivo flow data in our study is presented as semiquantitative with resolution of three-dimensional velocity vector in multiple slices. This gives timedependent, directional, and magnitude information about flow along the plane of the connection. We believe 
a descriptive account with or without the aid of instantaneous images of an inherently dynamic process falls far short of an accurate portrayal of flow. Moreover, in many of our patients flow disturbances were of a different nature in different cardiac phases and hence, no single instantaneous frame or series of frames would be representative of the complete flow characteristics. An animated display of such a dataset gives better understanding of flow characteristics to the viewer. Furthermore, data can be interactively viewed either in a loop format with speed controls or frame by frame advancement as determined by the viewer. Thus, an intuitive sense for the flow disturbances can be derived that is not achievable by any other means. We believe that our animated presentation of representative flow disturbances is a useful and currently unique method of sharing complex fluid dynamic information.

\section{Clinical Implications}

Ideally, for designing the optimal geometry of TCPC for conserving energy losses, direct in vivo assessment of pressure measurement and its correlation with energy losses should be done. However, because no method enables this, indirect means of assessing flow energetics are used. Our in vitro study used glass phantoms in which both pressure measurements and flow visualization were performed. The study patients yielded in vivo semiquantitative flow visualization. With comparative analysis of these methods, and keeping in mind anatomic space constraints, some cautious clinical implications can be made.

First, our and other studies [13] have shown the advantages of caval offsetting and, we believe, that within any given anatomic space constraints, offsetting should be incorporated as the first step in all TCPC designs. Whether the SVC should be offset more toward the left PA as in the extracardiac TCPC group or toward the right $\mathrm{PA}$ as in our intraatrial TCPC group, has not been determined by this study.

Second, if space permits, the TCPC should be flared open in all directions. If this is not possible due to space constraints then flaring should be done in such a fashion that the caval flows are not directed toward each other (Fig 9A). Thus, when the SVC is offset toward the left PA, the predominant flare should also be toward the left PA (Fig 9C).

Third, in the setting of bilateral SVCs, the two SVCs should each be preferentially flared toward the ipsilateral PA so as to direct blood peripherally into the pulmonary arteries. The ICC connection can be placed between the two SVC connections with again outward flaring to direct flow toward the branch PAs (Fig 9B). A similar approach can be adopted for bringing up hepatic venous drainage in the presence of interrupted IVC with azygos continuation. Such a design would allow hepatic venous blood distribution to both lungs and possibly prevent development of pulmonary arteriovenous malformation. On the basis of our in vivo and in vitro studies we would consider the design shown in Figure $9 \mathrm{C}$ as an optimal design. The SVC and the ICC are offset from each other. The SVC is flared in all directions but preferentially toward the left and the ICC flare is more toward the right. We believe that such a design would minimize interaction of flow and may be energy conserving.

\section{Limitations}

The nature of flow in the TCPC is complex and has cardiac and respiratory factors that influence it, as pointed out by Fogel and colleagues [14]. In our study, the impact of respiration on flow dynamics was not studied and is a limitation as the effects can be significant. Instead, in our study each phase was acquired at the nadir of respiratory cycle thus minimizing its effect. At this time our study is limited to a semiquantitative analysis of flow within the TCPC. From our acquired data a quantitative calculation of energy loss is possible. However, the quantitative methods need validation and we are in the process of establishing that validation. The complexity of flow disturbances in the TCPC may not be fully characterized by studying the flow only along coronal planes and in this regard our study is somewhat limited. Time constraints on the magnet prevented us from acquiring data in the axial planes along the TCPC pathway of our patients. Such an acquisition would have been useful for also confirming the flow disturbances noted in the coronal planes.

In conclusion, from this study we found that there is disordered flow in the inferior caval conduit of all our patients with TCPC. The flow dynamics at the cavopulmonary connection appear to be geometry dependent. Geometries that include caval offset and appropriate flaring appear to have favorable flow dynamics both in vivo and in vitro.

We thank Ginny Huber and Joycelyn Wade for their assistance in preparation of this manuscript and Kathy Murphy, RN, for helping with patient recruitment.

\section{References}

1. Mayer JE Jr, Bridges ND, Lock JE, Hanley FL, Jonas RA. Castenada. Factors associated with marked reduction in mortality for Fontan operations in patients with single ventricle. J Thorac Cardiovasc Surg 1992;103:444-52.

2. Bridges ND, Lock JE, Castaneda AR. Baffle fenstration with subsequent transcatheter closure: modification of the Fontan operation for patients at increased risk. Circulation 1990;82: 1681-9.

3. Laks H, Ardehali A, Grant PW, et al. Modification of the Fontan procedure: superior vena cava to left pulmonary artery connection and inferior vena cava to right pulmonary artery connection with adjustable atrial septal defect. Circulation 1995;91:2943-7.

4. Gale AW, Danielson GK, McGoon DC, Mair DD. Modified Fontan operation for univentricular heart and complicated congenital lesions. J Thorac Cardiovasc Surg 1979;78:831-8.

5. McElhinney DB, Petrossian E, Reddy VM, Hanley FL. Extracardiac conduit Fontan procedure without cardiopulmonary bypass. Ann Thorac Surg 1998;66:1826-8.

6. De Leval MR, Kilner P, Gewillig M, Bull C, McGoon DC. Total cavopulmonary connection: a logical alternative to atriopulmonary connection for complex Fontan operations. J Thorac Cardiovasc Surg 1988;96:682-95. 
7. Kim YH, Walker PG, Fontaine AA, Ensley AE, Oshinski J, et al. Hemodynamics of the Fontan procedures: an in vitro study. J Biomech Eng 1995;117:423-8.

8. Low $\mathrm{H}$, Chew $\mathrm{Y}$, Lee C. Flow studies on atriopulmonary and cavopulmonary connections of the Fontan operations for congenital heart defects. J Biomed Eng 1993;15:303-7.

9. Be'eri E, Maier SE, Landzberg MJ, Chung T, Geva T. In vivo evaluation of Fontan pathway flow dynamics by multidimensional phase-velocity magnetic resonance imaging. Circulation 1998;98:2873-82.

10. Sharma S, Goudy S, Walker P, et al. In vitro flow experiments for determination of optimal geometry of total cavopulmonary connection for surgical repair of children with functional single ventricle. J Am Coll Cardiol 1996;27:1264-9.

11. Ensley AE, Lynch P, Chatzimavroudis GP, Lucas C, Sharma $S$, Yoganathan AP. Toward designing the optimal total cavopulmonary connection: an in vitro study. Ann Thorac Surg 1999;68:1384-90.

12. Healy TM, Ensley AE, Ramuzat A, Sharma S, Yoganathan AP. Reconstruction and visualization of three-dimensional time dependent velocity fields obtained from magnetic resonance imaging. 1999 ASME Bioengineering Conference, Big Sky, MT, 1999.

13. Van Haesdonck JM, Mertens L, Sizaire R, et al. Comparison by computerized numeric modeling of energy losses in different Fontan connection. Circulation 1995;92(Suppl 2):322-6.
14. Fogel MA, Weinberg PM, Hoydu A, et al. The nature of flow in the systemic venous pathway measured by magnetic resonance blood tagging in patients having the Fontan operation. J Thorac Cardiovasc Surg 1996;114:1032-41.

15. Larado AC, Webber SA, Friehs I, del Nido PJ, Cape EG. Fluid dynamic comparison of intra-atrial and extracardiac total cavopulmonary connections. J Thorac Cardiovac Surg 1999; 117:697-704.

16. Hsia TY, Khambadkone S, Migliavacca F, Cohen G, Deanfield JE, de Leval MR. Effects of respiration and gravity on infra-diaphragmatic venous flow in normal and fontan patients [Abstract]. Circulation 1999;100(Suppl):I671.

17. Morgan VL, Grahm TP, Roselli RJ, Lorenz CH. Alterations in pulmonary artery flow patterns and shear stress determined with three-dimensional phase-contrast magnetic resonance imaging in Fontan patients. J Thorac Cardiovasc Surg 1997; 116:2 294-304.

18. Rebergen SA, Ottenkamp J, Doornbos J, Van Der Wall EE, Chin JGJ, De Roos A. Postoperative pulmonary flow dynamics after Fontan surgery: assessment with nuclear magnetic resonance velocity mapping. J Am Coll Cardiol 1993;21: 123-31.

19. Redington AN, Penny D, Shinebourne EA. Pulmonary blood flow after total cavopulmonary shunt. Br Heart J 1991;65: 213-7. 